\title{
Complete genome sequence of Anaerococcus prevotii type strain (PC1 $)$
}

Kurt LaButti ${ }^{1}$, Rüdiger Pukall ${ }^{2}$, Katja Steenblock ${ }^{2}$, Tijana Glavina Del Rio' ${ }^{1}$, Hope Tice ${ }^{1}$, Alex Copeland ${ }^{1}$, Jan-Fang Cheng', Susan Lucas ${ }^{1}$, Feng Chen ${ }^{1}$, Matt Nolan ${ }^{1}$, David Bruce ${ }^{1,3}$, Lynne Goodwin ${ }^{1,3}$, Sam Pitluck ${ }^{1}$, Natalia Ivanova ${ }^{1}$, Konstantinos Mavromatis ${ }^{1}$, Galina Ovchinniko$\mathrm{va}^{1}{ }^{1}$, Amrita Pati ${ }^{1}$, Amy Chen ${ }^{4}$, Krishna Palaniappan ${ }^{4}$, Miriam Land ${ }^{1,5}$, Loren Hauser ${ }^{1,5}$, YunJuan Chang 1,5, Cynthia D. Jeffries ${ }^{1,5}$, Patrick Chain ${ }^{1,6}$, Elizabeth Saunders ${ }^{1,3}$, Thomas Brettinn $^{1,3}$, John C. Detter ${ }^{1,3}$, Cliff Han ${ }^{1,3}$, Markus Göker ${ }^{2}$, Jim Bristow ${ }^{1}$, Jonathan A. Eisen ${ }^{1,7}$, Victor Markowitz ${ }^{4}$, Philip Hugenholtz ${ }^{1}$, Nikos C Kyrpides ${ }^{1}$, Hans-Peter Klenk², and Alla Lapidus $^{1 *}$

${ }^{1}$ DOE Joint Genome Institute, Walnut Creek, California, USA

${ }^{2}$ DSMZ - German Collection of Microorganisms and Cell Cultures GmbH, Braunschweig, Germany

${ }^{3}$ Los Alamos National Laboratory, Bioscience Division, Los Alamos, New Mexico, USA

${ }^{4}$ Biological Data Management and Technology Center, Lawrence Berkeley National Laboratory, Berkeley, California, USA

${ }^{5}$ Oak Ridge National Laboratory, Oak Ridge, Tennessee, USA

${ }^{6}$ Lawrence Livermore National Laboratory, Livermore, California, USA

${ }^{7}$ University of California Davis Genome Center, Davis, California, USA

*Corresponding author: Alla Lapidus

Keywords: Firmicutes, Clostridiales, 'Peptostreptococcaceae', Gram-positive, coccoid, human oral microflora, skin, non-motile, non-sporulating, anaerobic

\footnotetext{
Anaerococcus prevotii (Foubert and Douglas 1948) Ezaki et al. 2001 is the type species of the genus, and is of phylogenetic interest because of its arguable assignment to the provisionally arranged family 'Peptostreptococcaceae'. A. prevotii is an obligate anaerobic coccus, usually arranged in clumps or tetrads. The strain, whose genome is described here, was originally isolated from human plasma; other strains of the species were also isolated from clinical specimen. Here we describe the features of this organism, together with the complete genome sequence and annotation. This is the first completed genome sequence of a member of the genus. Next to Finegoldia magna, A. prevotii is only the second species from the family 'Peptostreptococcaceae' for which a complete genome sequence is described. The 1,998,633 bp long genome (chromosome and one plasmid) with its 1852 protein-coding and 61 RNA genes is a part of the Genomic Encyclopedia of Bacteria and Archaea project.
}

\section{Introduction}

Anaerococcus prevotii strain PC1 $1^{\mathrm{T}}$ (= DSM $20548=$ ATCC $9321=$ JCM 6508) is the type strain of the species and the type species of the genus [1]. Six strains of the species were characterized by Foubert and Douglas in 1948, originally designated as 'Micrococcus prevotii', but subsequently placed in the genus Peptococcus [2]. Based on a comparative study published by Ezaki et al. [3], the type strain of $P$. prevotii was then transferred to the genus 'Peptostreptococcus' and later on assigned to the novel genus Anaerococcus as A. prevotii [1]. The organism is a Gram-positive, anaerobic, indolenegative coccus. The major metabolic end product from metabolism of peptone-yeast-glucose (PYG) is butyric acid. A prevotii was provisionally assigned to the arranged family 'Peptostreptococcaceae' within the order Clostridiales, also designated as Family XI Incertae sedis [4]. Here we present a summary classification and a set of features for $A$. prevotii strain $\mathrm{PC}^{\mathrm{T}}$ together with the de- 
scription of the complete genomic sequencing and annotation.

\section{Classification and features}

Within the last few years, several changes occurred in the classification of the anaerobic Gram-positive cocci. There are currently five genera of anaerobic Gram-positive cocci which may be isolated from humans (Peptostreptococcus, Peptoniphilus, Parvimonas, Finegoldia, and Anaerococcus). Members of the species $A$. prevotii are frequently recovered from human clinical specimens such as vaginal discharges and ovarian, peritoneal, sacral or lung abscesses. In particular, $A$. prevotii was also described as a common isolate of the normal flora of skin, the oral cavity and the gut [3]. Historically the Grampositive anaerobic cocci were identified mainly by using phenotypic traits, but as shown by Song et al., this often led to the misidentification of $A$. vaginalis strains, which were mistakenly assigned to $A$. prevotii or A. tetradius [5]. Currently Genbank does not contain any16S rRNA sequences from cultivated strains that can be clearly linked to the spe- cies $A$. prevotii with over $95 \%$ gene sequence similarity. Recently, the temporal diversity of the human skin microbiome was analyzed using 16S rRNA gene phylotyping. It is noteworthy that several clones originated from different skin sites (gluteal crease, occiput, umbilicus, popliteal fossa, volar forearm). These isolates were taken from two patients and showed close relationships to A. prevotii [6]. No closely related isolates or uncultivated clones with more than 84\% 16S rRNA gene sequence identity are recorded from global ocean screenings and environmental samples (except for human skin).

Figure 1 shows the phylogenetic neighborhood of $A$. prevotii strain $\mathrm{PC}^{\mathrm{T}}$ in a $16 \mathrm{~S}$ rRNA based tree. The four 16S rRNA gene copies in the genome of strain $\mathrm{PC} 1^{\mathrm{T}}$ differ by up to 15 nucleotides from each other, and by up to 9 nucleotides from the previously published 16S rRNA sequence generated from strain CCUG 41932 (AF542232). The difference between the genome data and the reported 16S rRNA gene sequence is most likely due to sequencing errors in the previously reported sequence data.

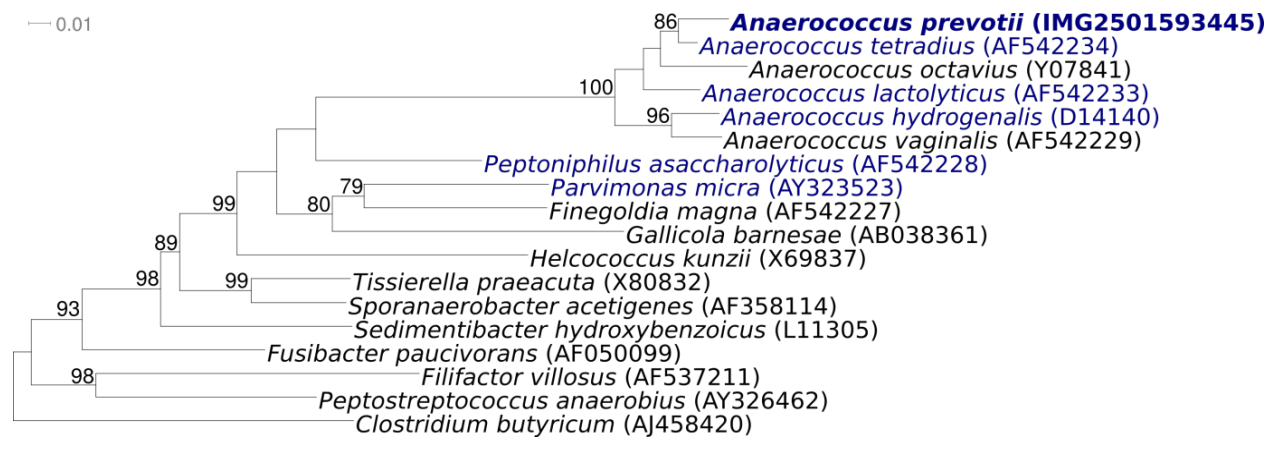

Figure 1. Phylogenetic tree highlighting the position of $A$. prevotii $\mathrm{PC}^{\top}$ relative to all type strains of the genus Anaerococcus and the type strains of all other genera within the family 'Peptostreptococcaceae' inferred from 1,302 aligned characters $[7,8]$ of the $16 \mathrm{~S}$ rRNA sequence under the maximum likelihood criterion [9]. Rooting was done with the type species of the genus Clostridium. The branches are scaled in terms of the expected number of substitutions per site. Numbers above branches are support values from 1,000 bootstrap replicates if larger than 60\%. Lineages with type strain genome sequencing projects registered in GOLD [10] are shown in blue, published genomes in bold.

A. prevotii $\mathrm{PC} 1^{\mathrm{T}}$ cells are Gram-positive and nonmotile(Table 1). Cells grown in PYG broth are 0.6-0.9 $\mu \mathrm{m}$ in diameter and occur in pairs, tetrads or irregular clumps or short chains (Figure 2). Colonies range from 0.5 to $2 \mathrm{~mm}$ in diameter on Columbia blood agar. Optimum temperature for growth is $37^{\circ} \mathrm{C}$. Strain $\mathrm{PC} 1^{\mathrm{T}}$ metabolizes peptones and amino acids and the major metabolic end product from PYG medium is butyric acid. Most species of the genus Anaerococcus ferment carbohydrates weakly. $A$. prevotii is proteolytic. $\alpha$-Glucosidase, $\alpha$-galactosidase, $ß$-glucuronidase and pyroglutamyl arylamidase activities are detectable [19,20]. Production of urease may vary among strains of the species. Most strains produce ammonia from threonine and serine [3] by deamination of the amino acids to pyruvate. A. prevotii is resistant to sodium polyanethol sulfonate [21], but susceptible to the penicillins [19]. 
Labuti, et al.

\section{Chemotaxonomy}

Cell wall amino acid analysis of strain PC1 ${ }^{\mathrm{T}}$ yielded peptidoglycan type A4 $\alpha^{\prime}$, composed of L-Lys-D-Glu [22], type A12.2 according to the DSMZ catalogue of strains. Cell wall sugars are glucose, glucosamine and galactose [22]. Major cellular fatty acid composition of the type strain was analyzed by Lambert and Armfield in 1979 [23] and by Ezaki et al. in 1983 [3], but the results of these studies are contradictory. No other chemotaxonomic data are available at present.

\section{Genome sequencing and annotation} Genome project history

This organism was selected for sequencing on the basis of its phylogenetic position, and is part of the Genomic Encyclopedia of Bacteria and Archaea project. The genome project is deposited in the Genomes OnLine Database [10] and the complete genome sequence has been deposited in GenBank. Sequence, finishing and annotation were performed by the DOE Joint Genome Institute (JGI). A summary of the project information is shown in Table 2

Table 1. Classification and general features of $A$. prevotii $\mathrm{PC}^{\top}$ in accordance with the MIGS recommendations [11]

\begin{tabular}{|c|c|c|c|}
\hline MIGS ID & Property & Term & $\begin{array}{l}\text { Evidence } \\
\text { code }\end{array}$ \\
\hline & \multirow{8}{*}{ Current classification } & Domain Bacteria & TAS [12] \\
\hline & & Phylum Firmicutes & TAS [13] \\
\hline & & Class Clostridia & TAS [4] \\
\hline & & Order Clostridiales & TAS [14] \\
\hline & & Family 'Peptostreptococcaceae' & TAS [4] \\
\hline & & Genus Anaerococcus & TAS [1] \\
\hline & & Species Anaerococcus prevotii & TAS [1] \\
\hline & & Type strain PC1 & TAS $[1,3]$ \\
\hline & Gram stain & positive & TAS [15] \\
\hline & Cell shape & coccoid & TAS [15] \\
\hline & Motility & nonmotile & TAS [15] \\
\hline & Sporulation & nonsporulating & TAS [15] \\
\hline & Temperature range & mesophile & TAS [15] \\
\hline & Optimum temperature & $37^{\circ} \mathrm{C}$ & TAS [15] \\
\hline & Salinity & growth in PYG $+6 \% \mathrm{NaCl}$ & TAS [16] \\
\hline \multirow[t]{3}{*}{ MIGS-22 } & Oxygen requirement & anaerobic & TAS [15] \\
\hline & Carbon source & unknown & \\
\hline & Energy source & peptones & TAS $[1,3]$ \\
\hline MIGS-6 & Habitat & human mouth, skin and vaginal microflora & TAS $[3,10]$ \\
\hline MIGS-15 & Biotic relationship & free living & NAS \\
\hline \multirow[t]{3}{*}{ MIGS-14 } & Pathogenicity & opportunistic infections & TAS [10] \\
\hline & Biosafety level & 2 & TAS [17] \\
\hline & Isolation & human plasma & TAS [3] \\
\hline MIGS-4 & Geographic location & not reported & \\
\hline $\begin{array}{l}\text { MIGS-5 } \\
\text { MIGS-4. } 1\end{array}$ & Sample collection time & not reported & \\
\hline MIGS-4.2 & Latitude - Longitude & not reported & \\
\hline MIGS-4.3 & Depth & not reported & \\
\hline MIGS-4.4 & Altitude & not reported & \\
\hline
\end{tabular}

Evidence codes - IDA: Inferred from Direct Assay (first time in publication); TAS: Traceable Author Statement (i.e., a direct report exists in the literature); NAS: Non-traceable Author Statement (i.e., not directly observed for the living, isolated sample, but based on a generally accepted property for the species, or anecdotal evidence). These evidence codes are available from the Gene Ontology project [18]. If the evidence code is IDA, then the property was directly observed for a live isolate by one of the authors or an expert mentioned in the acknowledgements. 
Table 2. Genome sequencing project information

\begin{tabular}{|c|c|c|}
\hline MIGS ID & Property & Term \\
\hline MIGS-31 & Finishing quality & $\begin{array}{l}\text { Finished } \\
\text { Three genomic libraries: two Sanger libraries - } 8 \mathrm{~kb} \\
\text { pMCL200 and fosmid pcc1 Fos - and one } 454 \text { pyrose- } \\
\text { quence standard library }\end{array}$ \\
\hline MIGS-29 & Sequencing platforms & ABI3730, 454 GS FLX, Illumina GA \\
\hline MIGS-31.2 & Sequencing coverage & 6.8 Sanger; 42.3 pyrosequence \\
\hline MIGS-30 & Assemblers & Newbler version 1.1.02.15, Arachne \\
\hline \multirow[t]{4}{*}{ MIGS-32 } & $\begin{array}{l}\text { Gene calling method } \\
\text { Genbank IDs } \\
\text { Genbank Dates of Release }\end{array}$ & $\begin{array}{l}\text { Prodigal, GenePRIMP } \\
\text { CP001708 (chromosome) } \\
\text { CP001709 (plasmid pAPRE01) } \\
\text { 4/28/2009 (chromosome) } \\
\text { 4/28/2009 (plasmid pAPRE01) }\end{array}$ \\
\hline & GOLD ID & Gc01089 \\
\hline & NCBI project ID & 29533 \\
\hline & Database: IMG-GEBA & 2501533213 \\
\hline \multirow[t]{2}{*}{ MIGS-13 } & Source identifier & DSM 20548 \\
\hline & Project relevance & Tree of Life, GEBA \\
\hline
\end{tabular}

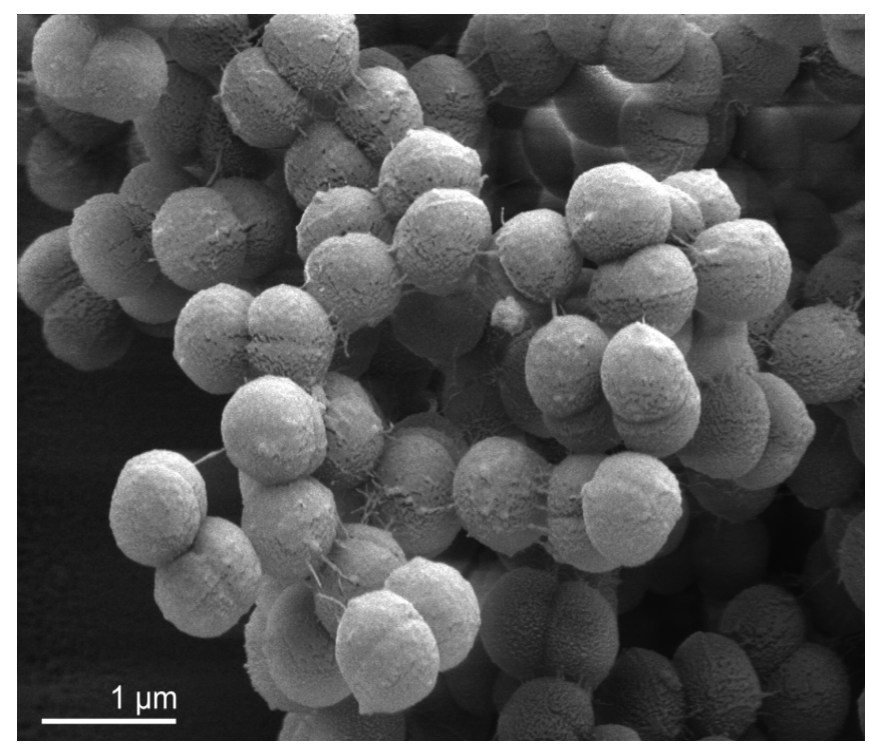

Figure 2. Scanning electron micrograph of $A$. prevotii $\mathrm{PC}^{\top}{ }^{\top}$ (M. Rohde, HZI Braunschweig)

\section{Growth condi tions and DNA isolation}

A. prevotii strain PC1 $1^{\mathrm{T}}$, DSM 20548, was grown anaerobically in DSMZ medium 104 [24] at $37^{\circ} \mathrm{C}$. DNA was isolated from 1-1.5 g of cell paste using Qiagen Genomic 500 DNA Kit (Qiagen, Hilden, Germany) following the instructions given by the manufacturer, but with a modified protocol for cell lysis, LALMP, according to Wu et al. [25].

\section{Genome sequencing and assembly}

The genome was sequenced using a combination of Sanger, 454 and Illumina sequencing platforms. All general aspects of library construction and sequencing can be found at the JGI web site. Reads produced by 454 Pyrosequencing were assembled using the Newbler assembler version 1.1.02.15 (Roche). Large Newbler contigs were broken into 2,196 overlapping fragments of $1,000 \mathrm{bp}$ and entered into the assembly as pseudo-reads. The sequences were assigned quality scores based on Newbler consensus q-scores with modifications to account for overlap redundancy and to adjust inflated q-scores. A hybrid 454/Sanger assembly was made using the Arachne assembler. Possible misassemblies were corrected and gaps between contigs were closed by custom primer walks from subclones or PCR products. A total of 66 Sanger finishing reads were produced. Illumina reads were used to improve the final consensus quality using an inhouse developed tool (the Polisher). The final assembly consisted of 18,576 Sanger and 464,157 Roche/454 reads. The error rate of the completed genome sequence is less than 1 in 100,000. Together all sequence types provided 49.1 coverage of the genome.

\section{Genome annotation}

Genes were identified using Prodigal [26] as part of the Oak Ridge National Laboratory genome annotation pipeline, followed by a round of manual curation using the JGI GenePRIMP pipeline [27]. The predicted CDSs were translated and used to search the National Center for Biotechnology Information (NCBI) nonredundant database, UniProt, TIGRFam, 
Labuti, et al.

Pfam, PRIAM, KEGG, COG, and InterPro databases. Additional gene prediction analysis and functional annotation was performed within the Integrated Microbial Genomes (IMG-ER) platform [28].

\section{Genome properties}

The genome is 1,998,633 bp long (chromosome and one circular plasmid) with a $35.6 \%$ GC content (Ta- ble 3). Of the 1,913 genes predicted, 1,852 were protein coding genes, and 61 were RNAs. A total of 46 pseudogenes were also identified, with $73.1 \%$ of the genes being assigned a putative function. The remaining genes were annotated as hypothetical proteins. The distribution of genes into COGs functional categories is presented in Figure 3 and Table 4.

Table 3. Genome Statistics

\begin{tabular}{lrr}
\hline Attribute & Value & \% of Total \\
\hline Genome size (bp) & $1,998,633$ & $100.00 \%$ \\
DNA Coding region (bp) & $1,815,671$ & $90.85 \%$ \\
DNA G+C content (bp) & 712,291 & $35.64 \%$ \\
Number of replicons & 2 & \\
Extrachromosomal elements & 1 & \\
Total genes & 1913 & $100.00 \%$ \\
RNA genes & 61 & $3.19 \%$ \\
rRNA operons & 4 & \\
Protein-coding genes & 1852 & $96.81 \%$ \\
Pseudo genes & 46 & $2.405 \%$ \\
Genes with function prediction & 1399 & $73.13 \%$ \\
Genes in paralog clusters & 231 & $12.08 \%$ \\
Genes assigned to COGs & 1421 & $74.28 \%$ \\
Genes assigned Pfam domains & 1428 & $74.65 \%$ \\
Genes with signal peptides & 337 & $17.62 \%$ \\
Genes with transmembrane helices & 467 & $24.41 \%$ \\
CRISPR repeats & 0 & \\
\hline
\end{tabular}
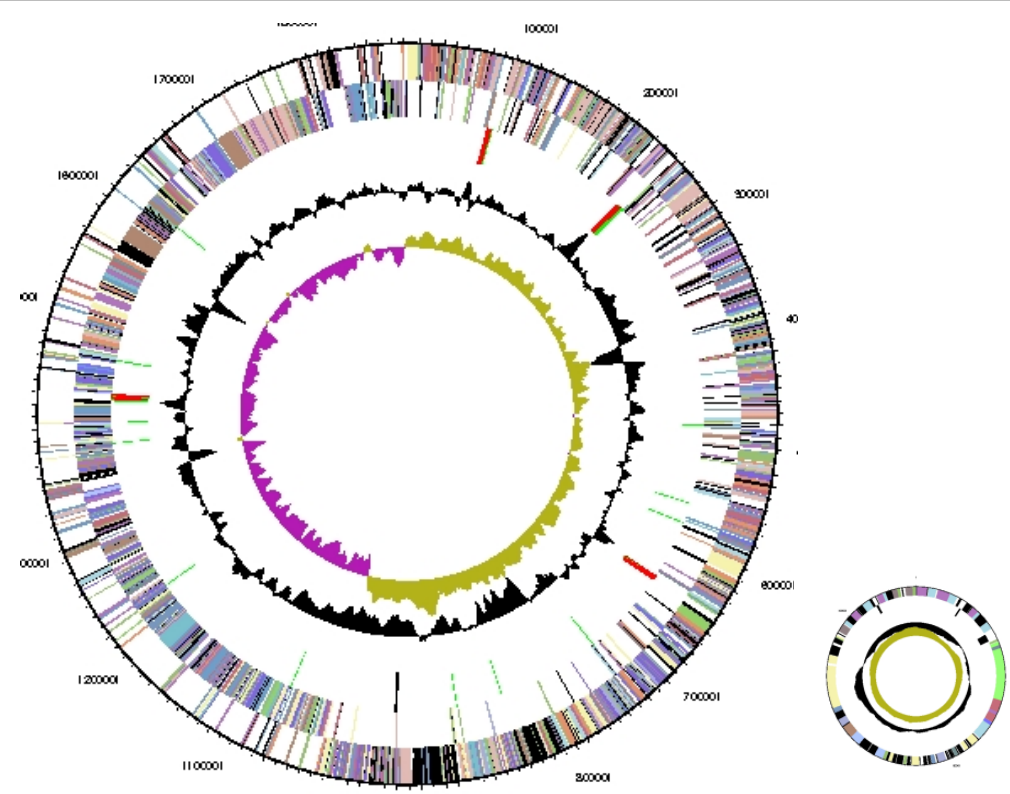

Figure 3. Graphical circular map of the genome. From outside to the center: Genes on forward strand (color by COG categories), Genes on reverse strand (color by COG categories), RNA genes (tRNAs green, sRNAs red, other RNAs black), GC content, GC skew. 


\begin{tabular}{lrrl}
\multicolumn{4}{l}{ Table 4. Number of genes associated with the 21 general COG functional categories } \\
\hline Code & Value & $\%$ & Description \\
\hline J & 133 & 7.2 & Translation, ribosomal structure and biogenesis \\
A & 0 & 0.0 & RNA processing and modification \\
K & 118 & 6.4 & Transcription \\
L & 105 & 5.7 & Replication, recombination and repair \\
$\mathrm{B}$ & 1 & 0.1 & Chromatin structure and dynamics \\
$\mathrm{D}$ & 20 & 1.1 & Cell cycle control, mitosis and meiosis \\
$\mathrm{Y}$ & 0 & 0.0 & Nuclear structure \\
$\mathrm{V}$ & 55 & 3.0 & Defense mechanisms \\
$\mathrm{T}$ & 43 & 2.3 & Signal transduction mechanisms \\
$\mathrm{M}$ & 69 & 3.7 & Cell wall/membrane biogenesis \\
$\mathrm{N}$ & 5 & 0.3 & Cell motility \\
$\mathrm{Z}$ & 0 & 0.0 & Cytoskeleton \\
$\mathrm{W}$ & 0 & 0.0 & Extracellular structures \\
$\mathrm{U}$ & 19 & 1.0 & Intracellular trafficking and secretion \\
$\mathrm{O}$ & 61 & 3.3 & Posttranslational modification, protein turnover, chaperones \\
$\mathrm{C}$ & 84 & 4.5 & Energy production and conversion \\
$\mathrm{G}$ & 144 & 7.8 & Carbohydrate transport and metabolism \\
$\mathrm{E}$ & 107 & 5.8 & Amino acid transport and metabolism \\
$\mathrm{F}$ & 61 & 3.3 & Nucleotide transport and metabolism \\
$\mathrm{H}$ & 56 & 3.0 & Coenzyme transport and metabolism \\
$\mathrm{I}$ & 37 & 2.0 & Lipid transport and metabolism \\
$\mathrm{P}$ & 102 & 5.5 & Inorganic ion transport and metabolism \\
$\mathrm{Q}$ & 9 & 0.5 & Secondary metabolites biosynthesis, transport and catabolism \\
$\mathrm{R}$ & 84 & 4.5 & General function prediction only \\
$\mathrm{S}$ & 118 & 6.4 & Function unknown \\
- & 431 & 23.3 & Not in COGs \\
\hline & & &
\end{tabular}

\section{Acknowledgements}

We would like to gratefully acknowledge the help of Susanne Schneider (DSMZ) for DNA extraction and quality analysis. This work was performed under the auspices of the US Department of Energy Office of Science, Biological and Environmental Research Program, and by the University of California, Lawrence Berkeley National Labo

\section{References}

1. Ezaki T, Kawamura Y, Li N, Li ZY, Zhao L, Shu S. Proposal of the genera Anaerococcus gen. nov., Peptoniphilus gen. nov. and Gallicola gen. nov. for members of the genus Peptostreptococcus. Int J Syst Evol Microbiol 2001; 51:1521-1528. PubMed

2. Foubert EL, Douglas HC. Studies on the Anaerobic Micrococci: I. Taxonomic Considerations. J Bacteriol 1948; 56:25-34.

3. Ezaki T, Yamamoto N, Ninomiya K, Suzuki S, Yabuuchi E. Transfer of Peptococcus indolicus, Peptococcus asaccharolyticus, Peptococcus prevotii, and Peptococcus magnus to the genus Peptostrep- ratory under contract No. DE-AC02-05CH11231, Lawrence Livermore National Laboratory under Contract No. DE-AC52-07NA27344, and Los Alamos National Laboratory under contract No. DE-AC02-06NA25396, as well as German Research Foundation (DFG) INST 599/1-1.

tococcus and proposal of Peptostreptococcus tetradius sp. nov. Int / Syst Bacteriol 1983; 33:683-698.

4. Garrity GM, Holt J. In: Garrity GM, Boone DR, Castenholz RW (2001). Taxonomic Outline of the Archaea and Bacteria. Bergey's Manual of Systematic Bacteriology, 2nd Ed. Volume 1 The Archaea, Deeply Branching and Phototrophic Bacteria. Springer-Verlag, New York pp 155-166

5. Song Y, Liu C, McTeague M, Vu A, Liu JY, Finegold SM. Rapid identification of Gram-positive anaerobic coccal species originally classified in the genus 
Labuti, et al.

Peptostreptococcus by multiplex PCR assays using genus- and species-specific primers. Microbiology 2003; 149:1719-1727. PubMed doi:10.1099/mic.0.26227-0

6. Grice EA, Kong HH, Conlan S, Deming CB, Davis J, Young AC, Bouffard GG, Blakesley RW, Murray PR, Green ED, et al. Topographical and temporal diversity of the human skin microbiome. Science 2009; 324:1190-1192. PubMed

7. Lee C, Grasso C, Sharlow MF. Multiple sequence alignment using partial order graphs. Bioinformatics 2002; 18:452-464. PubMed doi:10.1093/bioinformatics/18.3.452

8. Castresana J. Selection of conserved blocks from multiple alignments for their use in phylogenetic analysis. Mol Biol Evol 2000; 17:540-552. PubMed

9. Stamatakis A, Hoover P, Rougemont J. A rapid bootstrap algorithm for the RAxML web-servers. Syst Biol 2008; 57:758-771. PubMed doi:10.1080/10635150802429642

10. Liolios K, Mavromatis K, Tavernarakis N, Kyrpides NC. The Genomes On Line Database (GOLD) in 2007: status of genomic and metagenomic projects and their associated metadata. Nucleic Acids Res 2008; 36:D475-D479. PubMed doi:10.1093/nar/gkm884

11. Field D, Garrity G, Gray T, Morrison N, Selengut J, Sterk P, Tatusova T, Thomson N, Allen MJ, Angiuoli $\mathrm{SV}$, et al. The minimum information about a genome sequence (MIGS) specification. Nat Biotechnol 2008; 26:541-547. PubMed doi:10.1038/nbt1360

12. Woese CR, Kandler O, Wheelis ML. Towards a natural system of organisms: proposal for the domains Archaea, Bacteria, and Eucarya. Proc Natl Acad Sci USA 1990; 87: 4576-4579. PubMed doi:10.1073/pnas.87.12.4576

13. Skerman VBD, McGowan V, Sneath PHA. Approved list of bacterial names. Int J Syst Bacteriol 1980; 30: 225-230.

14. Prevot AR.. Dictionnaire des Bactéries Pathogènes. In: Hauduroy P, Ehringer G, Guillot G, Magrou J, Prevot AR, Rosset A, Urbain A, editors. 2nd ed. Paris 1953.

15. Douglas HC. Genus VI Peptococcus. Bergey's Manual of Determinative Bacteriology1957. p 474480.

16. Holdeman LV, Johnson J, Moore WEC. Genus Peptostreptococcus. In: Sneath P, editor. Bergey's
Manual of Systematic Bacteriology. Volume 2. Williams \& Wilkins, Baltimore 1986. p 1083-1092.

17. Biological Agents. Technical rules for biological agents www.baua.de TRBA 466.

18. Ashburner M, Ball CA, Blake JA, Botstein D, Butler $\mathrm{H}$, Cherry JM, Davis AP, Dolinski K, Dwight SS, Eppig JT, et al. Gene ontology: tool for the unification of biology. The Gene Ontology Consortium. Nat Genet 2000; 25:25-29. PubMed doi:10.1038/75556

19. Murdoch DA. Gram-positive anaerobic cocci. [PubMed]. Clin Microbiol Rev 1998; 11:81-120. PubMed

20. Ezaki T, Li N, Kaamura Y. The Anaerobic Grampositive cocci. The Prokaryotes. 3rd ed. Volume 4. New York: Springer 2006. p 795-808.

21. Song Y, Liu C, Finegold SM. Development of a flow chart for identification of gram-positive anaerobic cocci in the clinical laboratory. J Clin Microbiol 2007; 45: 512-516. PubMed doi:10.1128/JCM.01872-06

22. Schleifer $\mathrm{KH}$, Nimmermann E. Peptidoglycan types of strains of the genus Peptococcus. Arch Mikrobiol 1973; 93: 245-258. PubMed doi:10.1007/BF00412024

23. Lambert MA, Armfield AY. Differentiation of Peptococcus and Peptostreptococcus by gas-liquid chromatography of cellular fatty acids and metabolic products. J Clin Microbiol 1979; 10:464-476. PubMed

24. List of media used at DSMZ for cell growth: http://www.dsmz.de/microorganisms/media_list.php

25. Wu M, Hugenholtz P, Mavromatis K, Pukall R, Dalin E, Ivanova N, Kunin V, Goodwin L, Tindall BJ, et al. A phylogeny-driven genomic encyclopedia of Bacteria and Archaea. Nature (In press).

26. Anonymous. Prodigal Prokaryotic Dynamic Programming Genefinding Algorithm. Oak Ridge National Laboratory and University of Tennessee 2009. http://compbio.ornl.gov/prodigal.

27. Pati A, Ivanova N, Mikhailova N, Ovchinikova G, Hooper SD, Lykidis A, Kyrpides NC. GenePRIMP: A Gene Prediction Improvement Pipeline for microbial genomes. (Submitted) 2009.

28. Markowitz V, Mavromatis K, Ivanova N, Chen IM, Chu K, Palaniappan K, Szeto E, Anderson I, Lykidis A, Kyrpides N. Expert Review of Functional Annotations for Microbial Genomes. Bioinformatics 2009; 25: 2271-2278. PubMed doi:10.1093/bioinformatics/btp393 